\title{
What Gets Lost
}

\author{
Jeff Wiese, MD, MHM, FACP*
}

Tulane University Health Sciences Center, New Orleans, Louisiana.

This issue of the Journal of Hospital Medicine highlights an important contribution to the evolving state of graduate medical education (GME). The study assesses the relationship between attending physician workload and teaching effectiveness and patient safety. ${ }^{1}$

From the outset, it is important to note that although the focus of this study is on teaching on the wards, this is not necessarily synonymous with learning on the wards. Even if a busy service compromises a faculty's teaching on the wards, more patients on a service might augment a resident's learning on the wards, from patients, peers, active clinical decision making, and overall exposure to diversity of disease.

The independent variable in this study is intensity, with the presumption that the number of patients is proportional to intensity, as codified by the Accreditation Council for Graduate Medical Education (ACGME) regulations regarding caps for admissions and service size. However, are 10 single-organ chest pain patients the same intensity of 5 septic patients? The authors address this issue as much as possible by integrating "expected mortality" as a surrogate measure of intensity. Yet, given the heterogeneity of severity of illness even within a diagnosis, this too is likely to be an inaccurate measure of the true intensity of a service. Of course, such measures do not touch upon the "social intensity" that varies widely from patient to patient, which might be more time consuming and mentally exhausting than managing the diagnosis itself.

However, these limitations aside, this study's biggest contribution is that it raises the question that will define GME in the years to come, "How does learning fluctuate with service intensity?" The Yerkes-Dodson curve was published in 1908, defining the relationship between stress and performance (Figure 1). ${ }^{2}$ Many have interpreted the ACGME rules on admission caps and duty hours as being designed to make a kinder, gentler learning environment. However, as the curve suggests, optimizing service intensity (stress) is much more than just being nice; it is about optimizing performance, both in the way of patient care and learning. The question of how learning fluctuates with

*Address for correspondence and reprint requests: Jeff Wiese, MD, Infectious Professor of Medicine, Senior Associate Dean, Tulane University School of Medicine, 1430 Tulane Ave., New Orleans, LA 70112;

Telephone: 504-988-1143; Fax: 504-988-1143; E-mail: jwiese@tulane.edu

Received: December 8, 2015; Accepted: December 9, 2015 2016 Society of Hospital Medicine DOI 10.1002/jhm.2537 Published online in Wiley Online Library (Wileyonlinelibrary.com). service intensity might be better framed as, "What gets lost in the space as you move to the right of the optimal stress zone on the Yerkes-Dodson curve?"

Quality is first. This study correlates intensity with adverse events, and though there is a modest association, this likely underestimates the true magnitude of the problem. The measures in this study are documented adverse events, and are thus unlikely to capture the "near misses" that increase with heightened stress and intensity. Mistakes increase as mental bandwidth is insufficient to think through the consequences of each decision. "Slips"- things you know you need to do but forget to do-increase as the mind becomes distracted.

"Good work" is next. All hospitalists know that it is possible to get a patient in and out of the hospital, but it is also possible to do so with such poor quality that the patient comes right back. Csikszentmihalyi described the concept of "flow": the ability to become fully immersed in a task, concentrating on nothing except that task at hand. ${ }^{3}$ What comes from flow is good work. Achieving flow requires the time to engage in a task, but it also requires that the mind is not distracted by the worry of what else needs to be done. As service intensity increases, so does fragmentation and distractions, both of which are enemies to flow. Achieving flow also might have implications for teaching and learning: Does it matter how good the teacher is, or how often she teaches, if the residents are so distracted that they are not "mentally there" and ready to receive that teaching?

The presumption underlying all GME is that practice makes perfect. However, practice does not make perfect; perfect practice makes perfect. Furthermore, just because you were physically there for an experience, does not mean you actually experienced it. It is possible to be engaged in a patient encounter, and mentally drive right past it, missing the full implications of the experience that would have presumptively allowed for improvement. The difference between practice and perfect practice is contingent upon mentally "being there" and upon the ability to reflect upon that experience such that improvement is possible. However, experiencing the experience and reflection require time and mental bandwidth; both are diminished as you move to the right of the optimal zone. One of the central roles of the attending is to help learners fully experience the experience and reflect upon how things could have been done better. Though not specifically addressed by this study, one 


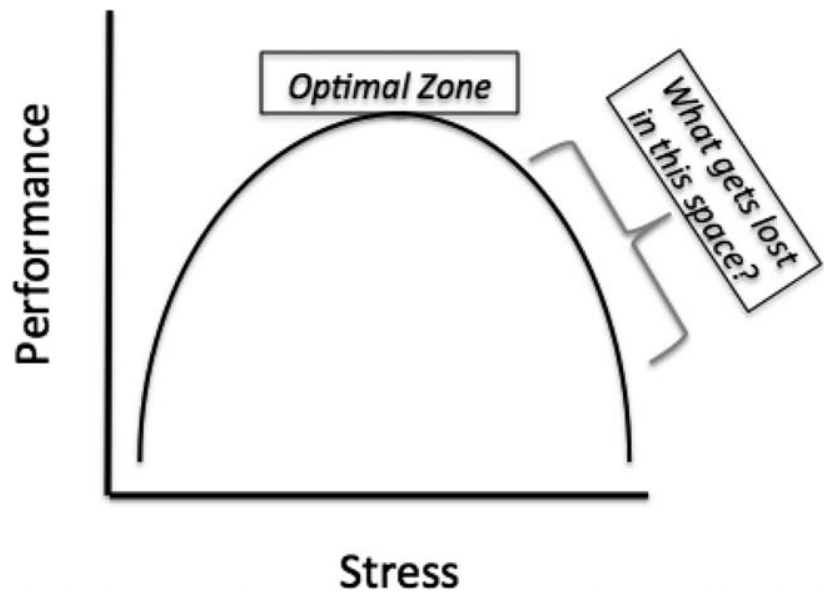

FIG. 1. The Yerkes-Dodson curve.

wonders if an attending on an intense teaching service has the time to provide that counsel, and even if they do, if the residents are in a mental position to receive it.

This study assesses the implications of a highly intense service on patient outcomes; what is not assessed are the implications for the future patients who will receive care from these residents. In Strangers to Ourselves, Wilson describes the "adaptive unconscious": the mind's ability to take routinely performed tasks and put them into an unconscious "hard drive" such that they can be completed at a later time without any conscious thought. ${ }^{4}$ It is adaptive, because it allows multitasking while doing rote activities. However, it is dangerous too, because once a rote task has been relegated to the adaptive unconscious, it is beyond the ability of the conscious mind to inspect and change it. The exponential consequence of imperfect practice is that the wrong thing done again and again settles into the adaptive unconscious, and there it will be for the rest of that resident's career. What is not specifically explored by this study, though nonetheless reasonable to assume, is that as a teaching service's intensity increases, the quality and frequency of attending feedback and resident selfreflection declines. The risk of a dysfunctional adaptive unconscious is inversely proportional to feedback and self-reflection.

So how do we redesign the inpatient GME experience to optimize performance? The architect tasked with designing an optimal learning environment for an inpatient service is tasked with addressing both ends of the Yerkes-Dodson curve. Too low of service intensity, residents lose out on exposure to diverse medical disease, and subsequent engagement in complex decision making requisite for developing their confidence and autonomy. Too high of service intensity, residents lose out on the teaching and feedback from their attendings, and the ability to truly experience and reflect upon the patients for whom they provide care. However, to do this effectively, the GME architect will need an accurate measure of inpatient intensity, something better than our current measures of duty hours and patient caps. Without that, it will be difficult to construct a learning environment that benefits not only the patients of today, but also the patients of tomorrow. One thing is for sure, the intensity of an inpatient service will only increase in the years to come, and the answer to the question of balancing intensity with learning, more than any other, will determine GME effectiveness. Achieving that balance will be a road of a thousand miles, but in raising this central question, this study gives us the first step.

\section{References}

1. Wingo MT, Halvorsen AJ, Beckman TJ, Johnson MG, Reed DA. Associations between attending physician workload, teaching effectiveness, and patient safety. J Hosp Med. 2016;11:169-173.

2. Yerkes RM, Dodson JD. The relation of strength of stimulus to rapidity of habit formation. J Comp Neurol Psychol. 1908;18:459-482.

3. Csikszentmihalyi M. Flow: The Psychology of Optimal Experience. New York, NY: Harper and Row; 1990.

4. Wilson T. Strangers to Ourselves: Discovering the Adaptive Unconscious. Cambridge, MA: Harvard University Press; 2002. 